\title{
The Application of Chinese Painting Elements in Modern Fashion Design
}

\author{
Huijuan Lai \\ Jiangxi Institute of Fashion Technology, Jiangxi, Nanchang, 330201 \\ 396526299@qq.com
}

Keywords: traditional Chinese painting; clothing design; application

\begin{abstract}
Chinese painting is the inheritance of Chinese national culture, with a long history and rich heritage, and occupies an important position in the world of art. Chinese painting with its unique artistic conception and artistically, contains rich Chinese traditional culture, applies Chinese painting to the modern clothing as a kind of clothing innovation design method.
\end{abstract}

\section{Introduction}

China has five thousand years of civilization history. In the transformation and precipitation of time, it has formed the unique artistic beauty of the East that attracts the attention of the world. Many artistic elements with ethnic characteristics are still in circulation today, such as: ink painting, painting , paper-cut, blue and white porcelain, Facebook, Chinese knots, plate buckles, auspicious patterns, etc. These artistic elements have become the national essence. The nationality is the world, and the clothing with national characteristics has a quiet temperament and profound charm. In the atmosphere of modern and rich social culture, it will always be a beautiful landscape, walking at the forefront of the world and leading the trend forward. Many designers will derive their inspiration from Chinese traditional patterns. Chinese paintings embody the concrete embodiment of the Chinese nation's cultural spirit and are well applied in clothing. The application of traditional patterns not only creates innovations in the design, but also adds up to the top five in China. Millennium culture agrees. We cannot forget our excellent traditional culture. It has been confirmed by history that it has been precipitated and accumulated through history. It is a highlight of our choice in design. It plays the finishing touch and shows the fashion moments of one nation after another.

\section{Cultural Characteristics and Formal Beauty of Chinese Painting}

\subsection{Cultural Characteristics of Chinese Painting.}

Unity. Chinese paintings and clothing should be unified in terms of style, color, style, etc.; pattern and social connotation should be unified; and the diversity of pattern expression should be unified. Decorative. Chinese painting is the decoration of clothing after the overall dress. Chinese painting not only plays a role in beautifying and emphasizing, but also plays a role in remedying defects. Decoration is human nature. As early as in ancient times, people used their leaves, animal bone ornaments, body paintings and shell feathers to decorate their bodies. Make up can emphasize or weaken some of the characteristics of the clothing shape and structure, life is not everyone's body is perfect, more or less there will be some defects, such as lean body, fat body, shoulder, slip Should shoulders, bulges, humps, chests, chicken breasts and other non-standard body characteristics, through the pattern design can be based on people's visual illusion characteristics can play a visual corrective effect, so that the body is perfect. Symbolic and allegorical. The pattern is one of the decorative elements in people's lives. It originates from life, embodies people's spiritual pursuit and longing for a better life. In ancient Chinese paintings, specific symbolic objects were used as the pursuit of an image. Peony symbolizes wealth; Ganoderma lucidum symbolizes longevity; fruit symbolizes harvest. 


\subsection{The Formal Rules of Chinese Painting.}

The pursuit of beauty is the nature of mankind. Especially when the level of spiritual civilization and material civilization is greatly improved, people have more and more pursuits of beauty. For all artistic creation, the use of formal beauty rules and the aesthetic sense of form Performance is essential. The beauty of symmetry and balance. Symmetry is also called homogeneous. According to the different angles of symmetry, there are generally three types of symmetry, vertical symmetry and reverse symmetry. For example, the portrait of the doorman in folk art in our country is approximately symmetrical. Balance means that the decorative elements can be freely arranged in a manner that is equal in shape, shape, or shape, etc. to obtain a psychological, visually stable, balanced form of construction. Its characteristic is that it is not limited by the axis of symmetry or symmetry, and the layout is relatively free. However, attention should be paid to the smoothness of the center of gravity of the screen. Such patterns have prominent themes, interspersed with freedom, good image stretching, flexible and varied movements, and strong sense of movement. .The beauty of lines. The line of Chinese painting also emphasizes mellowness, rhythm, and stiffness. Each flow should have a sense of mobility and power, giving people a sense of enthusiasm and a sense of vitality. The perfect combination of pen and ink, as the main expression language of Chinese painting, is an indispensable part of Chinese painting art. It expresses the author's aesthetic emotions by means of various abstract points, lines, renderings, and rubbings to extract the bones of all things. The ink-and-ink form of dot-line communication is the main abstract form of Chinese painting.

\section{The Application of Chinese Painting in Fashion Design}

Chinese painting is a shining point in the whole costume. It can well reflect the designer's style characteristics. Because of the limitation of the area and shape of the pattern in different parts of the garment, the pattern of expression must be appropriate, so this requires the designer to select the appropriate application form.

\subsection{Individual positioning pattern application.}

The positioning pattern in the garment is designed according to the specific size of the specific part of the garment. It is designed for different parts, collars, sleeves, front plackets, hem, back, shoulders, waist, buttocks, legs, etc. Form is the most widely used in traditional clothing. The traditional traditional folk costumes used in the men's design work have precisely applied positioning patterns. On the sleeves, use the form of painting to make reasonable typesetting according to the size of the area. At the front hem, use flowers and plants to draw flowers and plants according to the size of the area.

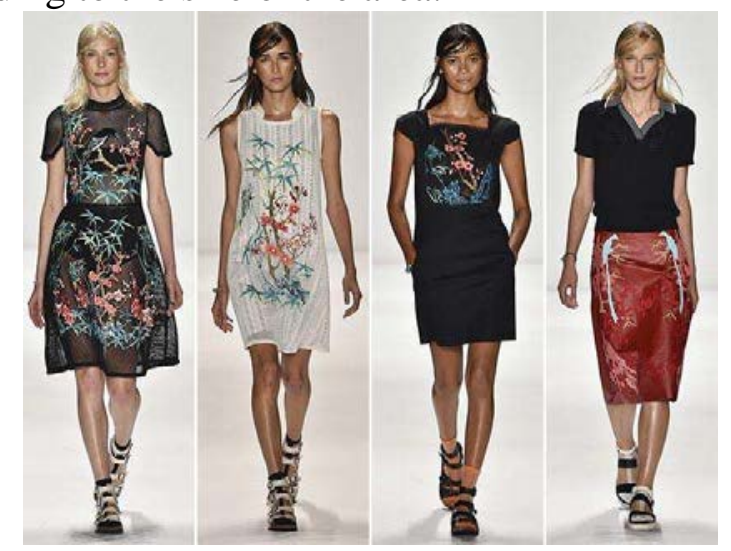

Fig.1 Positioning pattern application

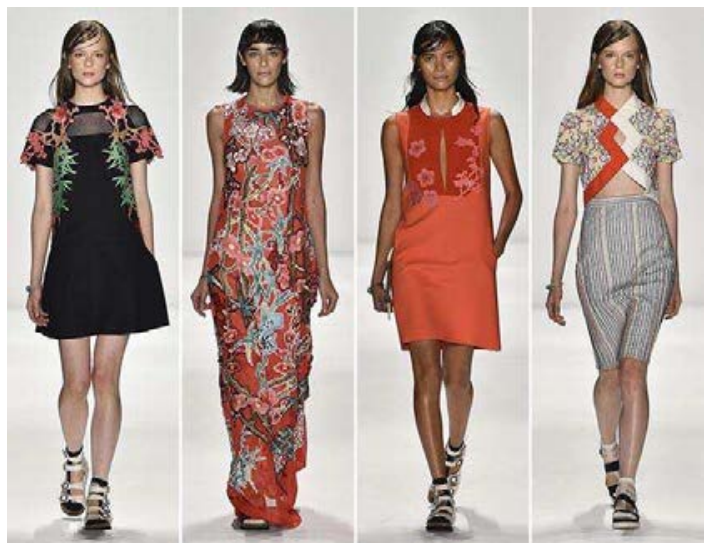

Fig.2 Plant pattern application

\subsection{Animal and Plant Patterns in Chinese Painting.}

In Chinese men's wear, Chinese painting uses blue and bamboo patterns of Chinese paintings, as well as flowers, birds, fruits, paintings, and lotus ink patterns. These patterns above all use the 
technique of drawing lines to embody the characteristics of the patterns. They also use some techniques of blooming to make the picture look more vivid, with a sense of rhythm and ambiguity, giving people a natural, relaxed and ethereal feeling. . The use of natural cotton and batik fabrics and batik fabrics on the fabrics makes the designs more unique and displays national style. Men's clothing, whether it is platemaking or craftsmanship, is demanding and delicate. The man's dress reflects his taste, and the correct dress makes them more confident and will increase their scores in their career. Men's wear does not have a lot of women's styles, nor does it have women's colors and patterns.

\subsection{Composition and Performance Techniques of Chinese Painting.}

The men's wear of national style clothing reflects another man's beauty and temperament. In the design, Chinese classical ink patterns were used, including blue, bamboo, lotus, and birds and flowers. Lan and bamboo are the four gentlemen in the flower. They have a certain symbolic significance in ancient times. Of course, in modern times, it does not lose its beautiful meaning. "The silt does not stain, and it is not the demon." This is where the lotus's character lies. Flowing love is not seduced by the secular world. The bird and fruit pattern is also reflected in the graduation design. The fruit symbolizes fruitfulness and symbolizes that we have a good harvest at work. The smudge method was used in the painting to make the picture look imaginary and lifelike. These designs are all drawn according to the style of the clothing in the design layout. Different styles and sizes of different parts are used to draw corresponding patterns so that the images do not look too full and will not appear as a space hole. In the drawing, different composition methods were used. Some use triangular composition, some use rectangular composition, of course, in the drawing will be based on the clothing's corner to draw. In the painting of clothes, the artistic conception of richness of the "leftover" of ink and wash is also applied. Leaving white is an important expression language of Chinese painting. The application of white space in clothing precisely reflects the cultural taste of human beings. Art and design are the same, they can all express the designer's wishes. Designers also use their subtleties to add luster to the work and to realize the value of these designs. In the designer's clothing works, the proper use of clothing will make the clothing look high-end atmosphere, and the use of bad will make the clothing look neither fish nor fowl, which requires the designer to have a considerable amount of accumulated experience or experience.

\subsection{The Combination of Chinese Painting and Fabric.}

The right choice of fabrics makes painting more convenient, and natural cotton and ink combine to give a Chinese retro feel. The retro thing is also one of the modern and popular elements. By using ancient elements to express people's nostalgia for the rush of time and the passage of time, it also shows its existence value and recognition by the society. The popular reincarnation verifies this, all things that are now popular will be outdated, and waiting for him to appear again is a reincarnation.
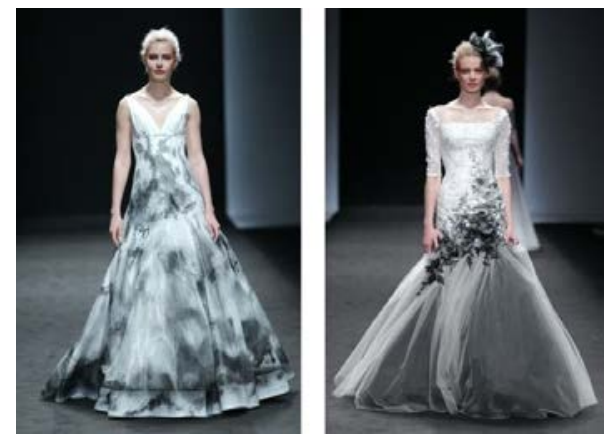

Fig.3 Ink rendering fabric

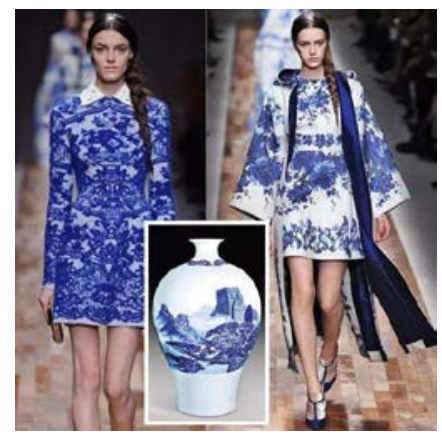

Fig.4 Blue and white pattern fabricl

\subsection{The Combination of Chinese Painting and Detail Elements.}

In the detailed design of the clothing, the collar design of the tunic suit, the disc buckle design of 
the Tang suit, and the raglan sleeve design were applied. The tunic has a strong symbol of political revolution and ideology. At that time, Zhongshan's impression of being loaded with people was conservative. However, the modern design of the reversible collar has made it more modern. Tang costume is one of the ancient costumes in China. The iconic plate buckle has been used as one of the popular elements of decorating life and expressing individuality. Not only has the domestic designer used the disc buckle, but even some of the foreign designers have learned the essence of it. Cleverly applied. In the graduation design, the elements of the modern suit and the clasp are combined to make it possess modern cultural features. The buckle uses cotton and linen fabrics and batik fabrics as the raw material of the buckles to achieve harmony and unity through reasonable cooperation. The design of the raglan sleeves makes the clothes more perfect, reflecting the gentleman's gentleness and style, reflecting the fashion sense in the whole series.

\section{Conclusion}

In short, in the design process, the entire series is echoed by combining Chinese painting with fabrics in a reasonable and orderly manner. The national style men's wear of Chinese painting shows gentle, stable, and strong cultural connotation of men. This is something that modern people lack. The positive energy conveyed by Chinese painting is also what we need to learn now. Chinese painting can cultivate the temperament of a person, and it can allow Mao Zedong's people to become calm and connotative.

\section{Reference}

[1] Shi Jing. The Application of Chinese Painting Elements in Custom Apparel Design [J] Popular Literature, 2014(19).

[2] Zhang Juan; Cui Rongrong. The application of traditional Chinese characters in the decoration of men's clothing design [J] Shandong Textile Economy, 2012 (02).

[3] Chen Tingting. The application of design elements in modern Chinese painting [J] Art Education, 2015(09).

[4] Wei Yulong. Embroidery innovation in modern clothing design and apply the research [D] jiangnan university, 2009 (6). 